\title{
Subretinal Hemorrhage after Photodynamic Therapy for Juxtapapillary Retinal Capillary Hemangioma
}

\author{
Takayuki Baba Masayasu Kitahashi Mariko Kubota-Taniai \\ Toshiyuki Oshitari Shuichi Yamamoto \\ Department of Ophthalmology and Visual Science, Chiba University Graduate \\ School of Medicine, Chiba, Japan
}

\section{Key Words}

Intravitreal bevacizumab · Juxtapapillary retinal capillary hemangioma · Photodynamic therapy · Subretinal hemorrhage

\begin{abstract}
A 75-year-old Japanese woman presented with a juxtapapillary retinal capillary hemangioma $(\mathrm{RCH})$ in her left eye. Twelve months after the initial examination, the size of the hemangioma had increased and the exudation from the $\mathrm{RCH}$ involved the macula. Her best-corrected visual acuity (BCVA) had decreased from 0.8 to 0.3 . A total of five intravitreal injections of bevacizumab (IVB; $1.25 \mathrm{mg}$ ) was given but the RCH did not respond. A photodynamic therapy (PDT) was done using multiple laser spots to avoid damaging the optic nerve head. After the first PDT, the subfoveal fluid was reduced but not completely gone. One week after the second PDT, a massive subretinal hemorrhage developed. The subretinal hemorrhage was successfully displaced by injecting intraocular sulfur hexafluoride $\left(\mathrm{SF}_{6}\right)$ gas. At the 3-year follow-up examination, no subretinal hemorrhage or fluid was observed at the macula and the BCVA remained at 0.05 . Our case was resistant to the combination of anti-vascular endothelial growth factor (VEGF) and PDT and had a rare massive subretinal hemorrhage. A further collection of RCH cases treated with anti-VEGF and PDT that would justify this treatment is necessary.
\end{abstract}

\section{Introduction}

A juxtapapillary retinal capillary hemangioma $(\mathrm{RCH})$ is a vascular tumor located at the border of the optic nerve head. It can occur as an isolated vascular abnormality or as a 
manifestation of von Hippel-Lindau (VHL) disease. Gass and Braunstein [1] described the characteristics of juxtapapillary RCHs and reported that photocoagulation was successful in some of their cases. Juxtapapillary RCH cases are diagnosed as being sessile or exophytic with the latter being more nodular with extension into the subretinal space. The extension into subretinal space results in the accumulation of subretinal fluid, which causes severe visual impairment when the fovea is involved.

Photocoagulation of the tumor has been widely accepted as a good treatment for juxtapapillary RCH $[2,3]$. However, the clinical outcome of photocoagulation has not been completely satisfactory because of the close proximity of the tumor to the optic disc and the progressive nature of the RCH. Recently, photodynamic therapy (PDT) has been reported to be an alternative method to treat juxtapapillary RCHs because PDT enables a more selective vascular occlusion and appears to be less damaging to the optic disc $[4,5]$. After the introduction of anti-vascular endothelial growth factor (VEGF) drugs, therapy with anti-VEGF drugs [6] or a combination of PDT with an intravitreal injection of antiVEGF drugs $[7,8]$ has been used to treat juxtapapillary RCH cases. Good results have been reported with these combined treatments.

However, complications, such as vitreous hemorrhages [9] and serous retinal detachments [10], have been reported after PDT for juxtapapillary RCH. We present a case of juxtapapillary $\mathrm{RCH}$ that developed a massive subretinal hemorrhage following multiple sessions of PDT and intravitreal bevacizumab (IVB). Such massive subretinal hemorrhage after PDT has rarely been reported.

\section{Case Report}

A 75-year-old Japanese woman presented with a complaint of blurred vision in her left eye. She had no significant ocular or systemic diseases. She also did not have any family history for ocular diseases. Her best-corrected visual acuity (BCVA) was $1.0 \mathrm{OD}$ and $0.8 \mathrm{OS}$, and the intraocular pressure was $9 \mathrm{mmHg}$ in the right eye and $8 \mathrm{mmHg}$ in the left eye. Slit-lamp examination showed no abnormalities except for mild cataracts in both eyes.

Ophthalmoscopy showed a one-disc-diameter, orange-colored region temporal and superior to the left optic disc suggesting a juxtapapillary hemangioma (fig. 1a). No other abnormalities were found in the retina of both eyes. Fluorescein angiography showed a diffuse fine capillary network that appeared to receive its blood supply from the ciliary and superficial retinal arteries (fig. 1b). The abnormal vascular network showed dye leakage in the late phase of fluorescein angiography (fig. 1c). These ophthalmoscopic and angiographic findings represented juxtapapillary neovascular membrane. Because the subretinal exudation was limited to the region around the RCH and did not extend to the fovea, the patient was followed without any treatment.

Twelve months after the initial examination, she noticed a decrease in the vision in her left eye and her BCVA had decreased to 0.3 . Ophthalmoscopy showed that the size of the hemangioma had increased and the exudation from the RCH involved the macula (fig. 1d, e). These findings were confirmed by optical coherence tomography (OCT) (fig. 1f).

After obtaining the patient's informed consent, an intravitreal injection of bevacizumab (IVB; 1.25 $\mathrm{mg}$ ) was given to reduce the vascular permeability of the RCH. Her BCVA in the left eye improved to 0.5 after a single IVB but dropped to 0.3 accompanied by a recurrence of the subretinal fluid at 6 months after the treatment. The patient received 4 more IVB treatments in the following 5 months. However, the exudation from the RCH remained and the subretinal fluid worsened the BCVA to 0.01.

After discussing other treatment options with the patient, we agreed to use PDT. The standard protocol of PDT which consisted of intravenous verteporfin at a dose of $6 \mathrm{mg} / \mathrm{m}^{2}$ body surface area and 
irradiation of diode laser $(689 \mathrm{~nm})$ at an intensity of $600 \mathrm{~mW} / \mathrm{cm}^{2}$ for $83 \mathrm{~s}\left(50 \mathrm{~J} / \mathrm{cm}^{2}\right)$ was used for each laser spot. To avoid damaging the optic nerve head, we used multiple laser spots of 2,300 and 3,400 $\mu \mathrm{m}$ that covered the entire hemangioma but did not irradiate the optic nerve head (fig. 2a, b). After the first PDT, the subfoveal fluid was reduced but not completely gone. We applied a second PDT with spot sizes of 2,500 and 4,500 $\mu \mathrm{m}$ based on the same strategy as the first session (fig. 2c, d). One week after the second PDT, a massive subretinal hemorrhage developed (fig. $3 \mathrm{a})$. The subretinal hemorrhage was successfully displaced by injecting intraocular sulfur hexafluoride $\left(\mathrm{SF}_{6}\right)$ gas. At the 3-year follow-up examination, no subretinal hemorrhage or fluid was observed at the macula and the BCVA remained at 0.05 (fig. 3b).

\section{Discussion}

The juxtapapillary RCH of our 75-year-old Japanese woman was diagnosed not to be due to VHL disease according to the diagnostic criteria for VHL disease established by Melmon and Rosen [11]. Our case did not have a family history of VHL disease, and there was no central nervous system or visceral hemangioma. In fact, solitary cases of RCH are more common than those in VHL disease [2].

Combined therapy using PDT and anti-VEGF drugs have been reported to be effective in treating solitary RCHs $[7,8]$. Pegaptanib, an anti-VEGF drug currently available, has been reported to decrease the retinal thickness and reduced the retinal hard exudates in some cases but did not stop the activity of RCH [6]. In a case treated by intravenous bevacizumab, there was only partial resolution of the exudation from the $\mathrm{RCH}$, and a recurrence of the subretinal fluid eventually occurred [10]. In our case, IVB was not totally effective in spite of multiple injections. As reported [6], the structure of the abnormal vessels of RCH is relatively mature and they might not depend on VEGF. If this is correct, then drugs targeting VEGF should be less effective for RCHs than for choroidal neovascularizations associated with age-related macular degeneration.

Massive subretinal hemorrhage is a rare complication associated with RCH. It occurred shortly after the second PDT session in our case. To protect the optic disc from irradiation and to cover the whole area of the hemangioma, we used multiple laser spots. Even though we designed our treatment to minimize overlapping areas, some parts of the hemangioma received a greater amount of energy than usual. We believe that this might have been the cause of the uneven occlusion of vessels of the tumor, and the vulnerable vessels after the first PDT hemorrhaged when they were reperfused. Consideration should be given to use reduced-fluence PDT when multiple PDT spots are applied.

In conclusion, we reported a patient with a juxtapapillary $\mathrm{RCH}$ who had a rare massive subretinal hemorrhage after multiple treatments with IVB and PDT. Although the macular edema was completely resolved, the final visual acuity was poor. Thus, our case was resistant to the combination of anti-VEGF and PDT. A further collection of RCH cases treated with anti-VEGF and PDT that would justify this treatment is necessary. 

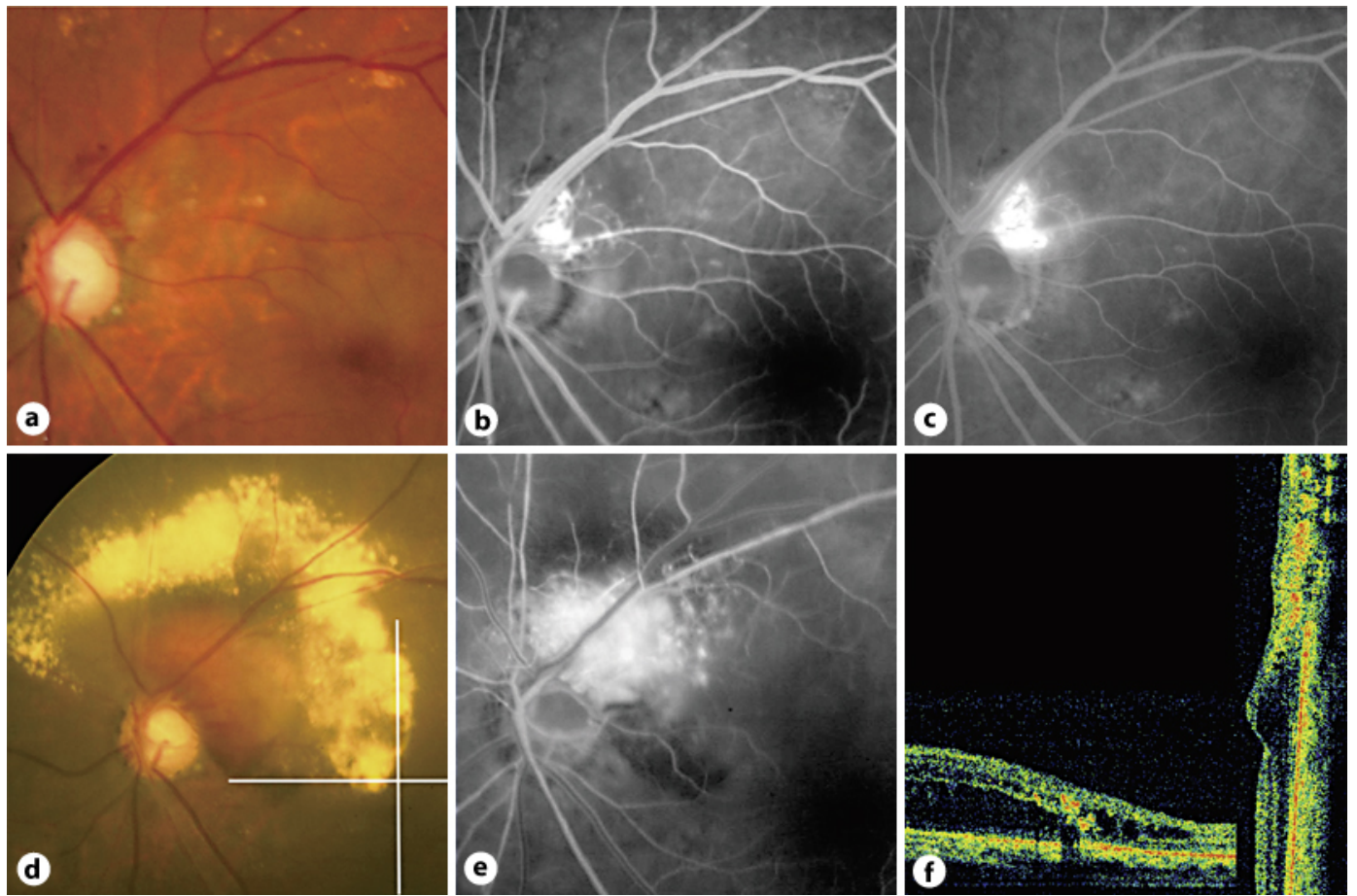

Fig. 1. a A juxtapapillary RCH can be seen at the temporal superior border of the optic disc. The patient's visual acuity was 0.8 . b Early-phase fluorescein angiogram showing fine network of vessels in the hemangioma. c Late-phase fluorescein angiogram showing dye leakage from the RCH. d One year after the initial presentation, the juxtapapillary $\mathrm{RCH}$ has significantly increased in size, and subretinal fluid and exudates are present surrounding the hemangioma. The visual acuity has dropped to 0.3. e Early-phase fluorescein angiogram showing a vascular-rich tumor growing outward from the optic disc. $\mathbf{f}$ Tomographic images shown by the scan lines in $\mathbf{d}$. The images show an accumulation of subretinal fluid extending to the macula. The deposition of hard exudates and intraretinal edema can also be seen. 

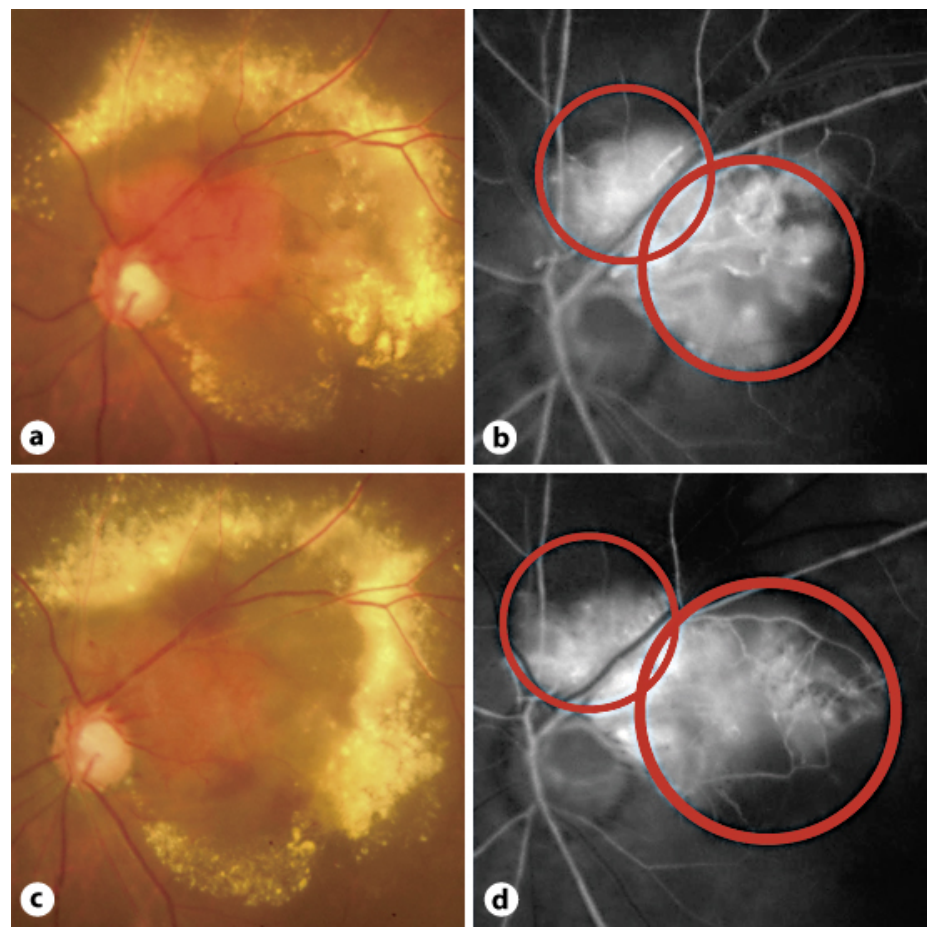

Fig. 2. a Photograph of RCH before the first PDT. The orange-colored lesion with prominent exudation was not reduced by multiple intravitreal bevacizumab and patient's visual acuity was 0.01 . b The red circles indicate the area where PDT was applied. To avoid the adverse effects to the optic disc, two laser spots of 2,300 and 3,400 $\mu \mathrm{m}$ were used. c After the first session of PDT, the RCH was still active showing further extension of the vascular lesion. $\mathbf{d}$ The red circles indicate the site of the PDT spots applied at the second PDT. Two laser spots of 2,500 and 4,500 $\mu \mathrm{m}$ were used.
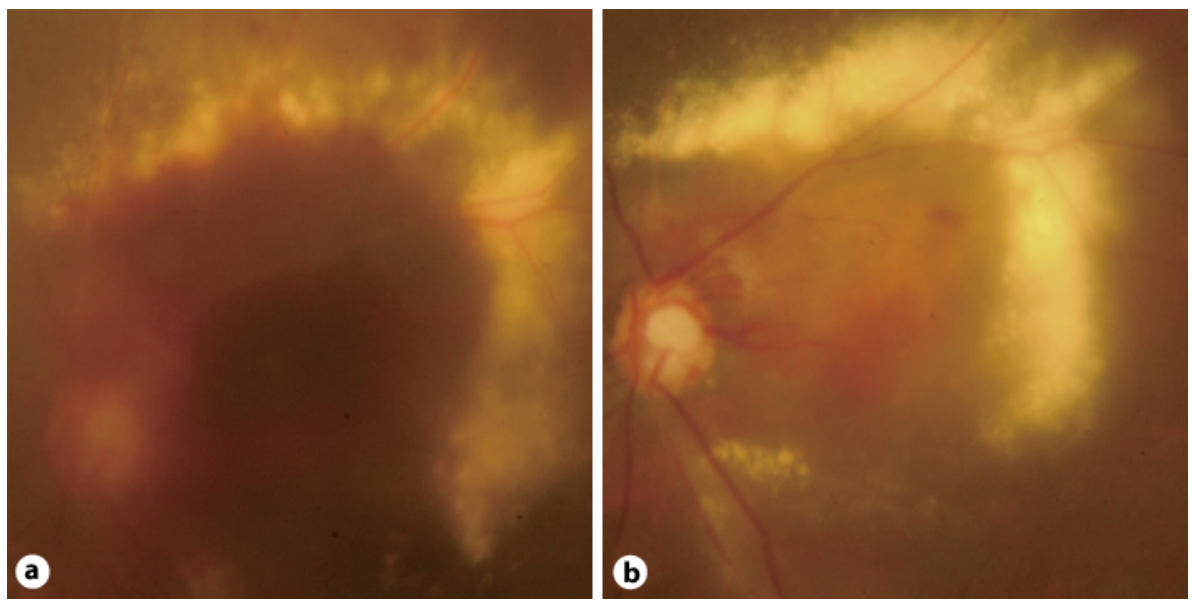

Fig. 3. a A massive subretinal hemorrhage including the macula developed 7 days after the second PDT. b After a pneumatic displacement using sulfur hexafluoride gas, the hemorrhage disappeared at the macula. The RCH showed regression and the subretinal fluid decreased. The visual acuity was 0.05 at the final visit. 


\section{References}

1 Gass JD, Braunstein R: Sessile and exophytic capillary angiomas of the juxtapapillary retina and optic nerve head. Arch Ophthalmol 1980;98:1790-1797.

$\longrightarrow 2$ McCabe CM, Flynn HW Jr, Shields CL, Shields JA, Regillo CD, McDonald HR, Berrocal MH, Gass JD, Mieler WF: Juxtapapillary capillary hemangiomas. Clinical features and visual acuity outcomes. Ophthalmology 2000;107:2240-2248.

-3 Singh AD, Nouri M, Shields CL, Shields JA, Perez N: Treatment of retinal capillary hemangioma. Ophthalmology 2002;109:1799-1806.

4 Golshevsky JR, O’Day J: Photodynamic therapy in the management of juxtapapillary capillary haemangiomas. Clin Experiment Ophthalmol 2005;33:509-512.

5 Sachdeva R, Dadgostar H, Kaiser PK, Sears JE, Singh AD: Verteporfin photodynamic therapy of six eyes with retinal capillary haemangioma. Acta Ophthalmol 2010;88:e334-e340.

-6 Dahr SS, Cusick M, Rodriguez-Coleman H, Srivastava SK, Thompson DJ, Linehan WM, Ferris FL 3rd, Chew EY: Intravitreal anti-vascular endothelial growth factor therapy with pegaptanib for advanced von HippelLindau disease of the retina. Retina 2007;27:150-158.

7 Ziemssen F, Voelker M, Inhoffen W, Bartz-Schmidt KU, Gelisken F: Combined treatment of a juxtapapillary retinal capillary haemangioma with intravitreal bevacizumab and photodynamic therapy. Eye (Lond) 2007;21:1125-1126.

$\checkmark 8$ Mennel S, Meyer CH, Callizo J: Combined intravitreal anti-vascular endothelial growth factor (Avastin) and photodynamic therapy to treat retinal juxtapapillary capillary haemangioma. Acta Ophthalmol 2010;88:610613.

-9 Reynolds SA, Shechtman D, Falco L: Complex juxtapapillary capillary hemangioma: a case report. Optometry 2008;79:512-517.

10 von Buelow M, Pape S, Hoerauf H: Systemic bevacizumab treatment of a juxtapapillary retinal haemangioma. Acta Ophthalmol Scand 2007;85:114-116.

11 Melmon KL, Rosen SW: Lindau's disease. Review of the literature and study of a large kindred. Am J Med 1964;36:595-617. 